А. Ф. Аникеенко, Т. А. Машорипова

Белорусский государственный технологический университет

\title{
ИССЛЕДОВАНИЕ ВЛИЯНИЯ ПАРАМЕТРОВ УПРУГИХ ЭЛЕМЕНТОВ НОВОЙ КОНСТРУКЦИИ СВЕРЛИЛЬНОГО ИНСТРУМЕНТА НА ГЛУБИНУ СВЕРЛЕНИЯ ЛДсТП
}

В статье описывается новая конструкция сверлильного инструмента с применением упругих элементов для сверления сквозных отверстий в ламинированных древесностружечных плитах. Данная конструкция позволяет механическим путем изменять скорость подачи непосредственно во время обработки ламинированной древесностружечной плиты, тем самым предотвращая появление сколов на поверхности хрупкого слоя (ламината) плиты.

Конструкция предполагает применение пружин сжатия с разными коэффициентами жесткости в сверлильном инструменте. Во время внедрения сверла в твердую ламинированную часть плиты снижается скорость подачи за счет срабатывания пружин, на которые в это время подается нагрузка.

Достоинства конструкции: при уменьшении скорости подачи образование сколов на входе и выходе ламинированной плиты минимально. Изменение скорости подачи происходит не на программном уровне, что влечет за собой написание более простой программы обработки заготовки; простоту изготовления конструкции; малые габариты конструкции.

За счет сжатия упругих элементов происходит уменьшение скорости подачи и ограничивается углубление сверлильного инструмента в обрабатываемый материал. В связи с этим в данной работе был проведен расчет глубины просверливаемого отверстия в зависимости от жесткости упругих элементов.

Ключевые слова: конструкция, совершенствование, древесностружечная плита, сверление, сверло.

Для цитирования: Аникеенко А. Ф., Машорипова Т. А. Исследование влияния параметров упругих элементов новой конструкции сверлильного инструмента на глубину сверления Л-ДсТП // Труды БГТУ. Сер. 1, Лесное хоз-во, природопользование и перераб. возобновляемых ресурсов. 2021. № 2 (246). С. 333-339.

\section{A. F. Anikeenko, T. A. Mashoripova \\ Belarusian State Technological University \\ INVESTIGATION OF THE INFLUENCE OF PARAMETERS \\ OF ELASTIC ELEMENTS OF A NEW DRILLING TOOL DESIGN ON THE DRILLING DEPTH OF LDSTP}

The article describes a new design of a drilling tool with the use of elastic elements for drilling through holes in laminated chipboard. This design allows you to mechanically change the feed rate directly during the processing of laminated chipboard, thereby preventing the appearance of chips on the surface of the brittle layer (laminate) of the board.

The design involves the use of compression springs with different stiffness coefficients in the drilling tool. During the introduction of the drill into the solid laminated part of the plate, the feed rate decreases due to the actuation of the springs, which are loaded at this time.

Advantages of the design: with a decrease in the feed rate, the formation of chips at the inlet and outlet of the laminated board is minimal. The change in the feed rate does not occur at the program level, which entails writing a simpler program for processing the workpiece; simplicity of manufacturing the structure; small dimensions of the structure.

Due to the compression of the elastic elements, the feed rate is reduced and the deepening of the drilling tool into the processed material is limited. In this regard, in this work, the depth of the drilled hole was calculated depending on the stiffness of the elastic elements.

Key words: design, improvement, particle board, drilling, drill.

For citation: Anikeenko F. A., Mashoripova T. A. Investigation of the influence of parameters of elastic elements of a new drilling tool design on the drilling depth of LDsTP. Proceedings of BSTU, issue 1, Forestry. Nature Management. Processing of Renewable Resources, 2021, no. 2 (246), pp. 333-339 (In Russian). 
Введение. На процесс резания древесины и древесных материалов оказывает влияние много факторов, среди которых можно выделить три основные группы:

1) факторы, относящиеся к исследуемому материалу (физико-механические свойства ламинированной древесностружечной плиты);

2) факторы, относящиеся к режущему инструменту (геометрические параметры сверла, углы резания, марка стали и пр.);

3) режимы резания или обработки (скорость главного движения, скорость подачи) [1-6].

Важными характеристиками инструмента, влияющими на качество обработки, силы и мощность резания, являются угол наклона винтовой канавки $\varepsilon$, угол подъема винтовой канавки $\tau$, диаметр сверла $D$ и угол при вершине $2 \varphi$ (рис. 1 ).

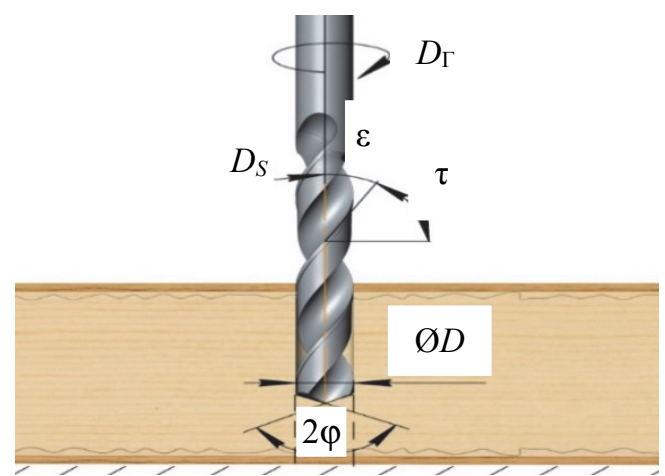

Рис. 1. Схема обработки сверлением

Цель исследования - изучить влияние параметров упругих элементов нового сверлильного инструмента на глубину сверления ламинированной древесностружечной плиты и на изменение скорости подачи. Задачи исследования:

1) составить методику расчета влияния жесткости упругих элементов на глубину сверления и изменение скорости подачи;

2) произвести расчеты для получения численных значений глубины сверления.

Объект исследования - сверло. Предмет исследования - глубина сверления.

Основная часть. Существуют различные методики проведения экспериментов по изучению свойств дереворежущего инструмента. Но большинство из них охватывает не более одного исследуемого варьируемого параметра, влияющего на интересующий нас показатель - качество обработанной поверхности.

При сверлении древесных материалов наиболее распространенной конфигурацией режущей части сверла являются коническая заточка и форма с подрезателями и направляющим центром. При этом при второй форме сверло имеет четыре режущих элемента: две главные режущие кромки и два подрезателя [7-9].
Существующие сверлильные инструменты для обработки древесины твердых и мягких пород, плитных материалов, имеют один существенный недостаток: предназначены для обработки только конкретного вида материала на определенных технологических режимах [10-15].

В связи с вышесказанным было спроектирован сверлильный инструмент, конструкция которого предполагает применение пружин сжатия с разными коэффициентами жесткости в сверлильном инструменте. Во время внедрения сверла в твердую ламинированную часть плиты снижается скорость подачи за счет срабатывания пружин, на которые в это время подается нагрузка.

На основании рассмотренных конструкций сверлильный инструмент с применением упругих элементов является наиболее оптимальным вариантом. Это достигается за счет простоты конструкции и меньших габаритов.

На рис. 2 представлена расчетная схема пружин.

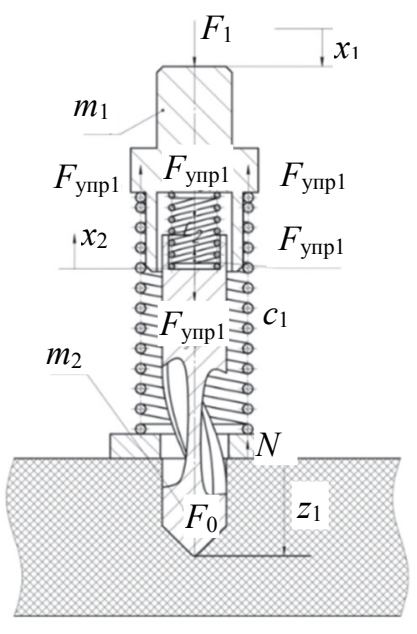

Рис. 2. Расчетная схема

Для решения составим следующие дифференциальные уравнения:

$$
\begin{gathered}
m_{1} \ddot{x}_{1}=F-c_{1} x_{2} ; \\
m_{2}\left(\ddot{x}_{1}-\ddot{x}_{2}\right)=c_{1} x_{2}-F_{O C},
\end{gathered}
$$

где $F$ - тяговое усилие, $\mathrm{H} ; c_{1}-$ коэффициент жесткости внешней пружины, Н/м; $x_{1}$ - деформация внешней пружины, м; $x_{2}-$ деформация внутренней пружины, м; $m_{1}$ - масса оправы $\left(m_{1}=0,275\right.$ кг); $m_{2}-$ масса сверла $\left(m_{2}=0,05\right.$ кг).

Далее произведем расчет тягового усилия.

Общая формула тягового усилия

$$
F>\sum F_{C} .
$$

Или формула (4) будет имеет вид

$$
F=\alpha \sum F_{C},
$$

где $F_{\mathrm{C}}$ - сумма сил сопротивления, $\mathrm{H} ; \alpha$ - коэффициент запаса. 
Исходя из расчетной схемы, сумма сил сопротивления будет равна

$$
\sum F_{C}=F_{S}-F_{\text {упр1 }}-F_{\text {упр } 2},
$$

где $F_{\text {упр1 }}$ и $F_{\text {упр2 }}-$ силы упругости внутренней и внешней пружины соответственно.

Расчетная схема представлена на рис. 3.

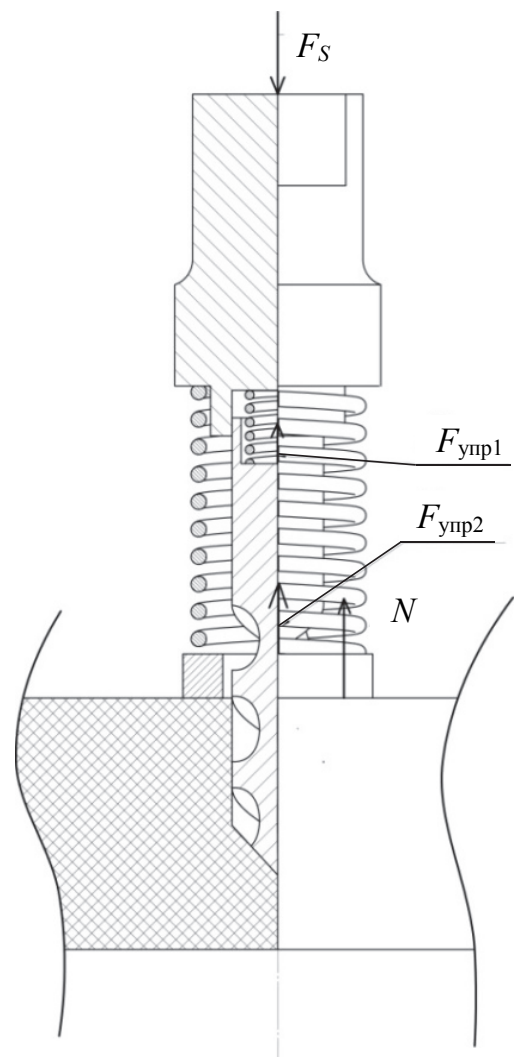

Рис. 3. Расчетная схема тягового усилия

Коэффициент запаса $\alpha=1,3-1,5$. В расчетах принимаем $\alpha=1,4$.

$$
F=1,4 \cdot 50,76=71,06 \mathrm{H} .
$$

Коэффициент жесткости пружин

$$
c_{1}=\frac{G \cdot d_{D}^{4}}{8 \cdot d_{F}^{3} \cdot n},
$$

где $G$ - модуль сдвига, ГПа; $d_{D}$ - диаметр проволоки, м; $d_{F}-$ диаметр завивки, м; $n$ - число витков.

Модуль сдвига $G=82$ ГПа для стали 60С2А. Диаметр проволоки $d_{D}=0,003$ м, диаметр завивки $d_{F}=0,022$ м, Число витков $n=10$.

$$
c_{1}=\frac{82 \cdot 10^{9} \cdot 0,003^{4}}{8 \cdot 0,022^{3} \cdot 10}=7797,1 \mathrm{H} / \mathrm{M} .
$$

Выразим из (1) $\ddot{x}_{1}$ и подставим в (2):

$$
m_{2}\left(\frac{F-c_{1} x_{2}}{m_{1}}-\ddot{x}_{2}\right)=c_{1} x_{2}-F_{0} \text {. }
$$

Преобразуем выражение (7), поделив правую и левую части на $m_{2}$ :

$$
\ddot{x}_{2}+c_{1} x_{2} \frac{m_{1}+m_{2}}{m_{1} m_{2}}=\frac{F_{0}}{m_{2}}+\frac{F}{m_{1}} .
$$

Для удобства записи произведем замену:

$$
\mu=\frac{m_{1} m_{2}}{m_{1}+m_{2}} ; K=\frac{F_{0}}{m_{2}}+\frac{F}{m_{1}} .
$$

Масса оправы равна $m_{1}=0,275$ кг, масса сверла $m_{2}=0,05$ кг. Осевая сила $F_{0}=159,07 \mathrm{H}$, тяговое усилие составляет $F=71,06 \mathrm{H}$.

$$
\begin{gathered}
\mu=\frac{0,275 \cdot 0,05}{0,275+0,05}=0,042, \\
K=\frac{159,07}{0,05}+\frac{71,06}{0,275}=3484 .
\end{gathered}
$$

Тогда выражение (8) примет вид

$$
\ddot{x}_{2}+\frac{c_{1}}{\mu} x_{2}=K \text {. }
$$

Решением данного уравнения будет являться выражение

$$
x_{2}=A \cdot \cos \theta k_{2} t+B \cdot \sin k_{2} t+\frac{K}{c_{1}} \mu,
$$

где $k_{2}$ - частота колебаний, $\mathrm{c}^{-1}$.

Частоту колебаний найдем из формулы

$$
k_{2}=\sqrt{\frac{c_{1}}{\mu}} .
$$

Коэффициент жесткости внешней пружины $c_{1}=7797,1 \mathrm{H} / \mathrm{M}, \mu=0,042$

$$
k_{2}=\sqrt{\frac{7797,1}{0,042}}=430 \mathrm{c}^{-1} .
$$

Для определения константы $A$ продифференцируем выражение (10):

$$
\dot{x}_{2}=-A k_{2} \sin k_{2} t+B k_{2} \cos k_{2} t .
$$

Начальные условия: $\dot{x}_{2}=0 ; x_{2}=0 ; t=0$.

Тогда из (10) и (11) следует, что

$$
A=-\frac{K}{c_{1}} \mu ; B=0 .
$$

Таким образом, с учетом найденных констант, выражение (9) примет вид

$$
x_{2}=\frac{K}{c_{1}} \mu\left(1-\cos k_{2} t\right) \text {. }
$$


Подставив выражение (13) в (2), получим

$$
m_{1} \ddot{x}_{1}=F-K \mu\left(1-\operatorname{cosk}_{2} t\right) .
$$

Разделим правую и левую части на $m_{1}$ :

$$
\ddot{x}_{1}=\frac{F-K \mu}{m_{1}}+\frac{K \mu}{m_{1}} \cos k_{2} t .
$$

Для определения $\dot{x}_{1}$ проинтегрируем выражение (15):

$$
\dot{x}_{1}=\int_{0}^{t} \frac{F-K \mu}{m_{1}} d t+\int_{0}^{t} \frac{K \mu}{m_{1}} \cos k_{2} t d t .
$$

Получим

$$
\dot{x}_{1}=\frac{F-K \mu}{m_{1}} t+\frac{K \mu}{m_{1} k_{2}} \sin k_{2} t+C_{1} .
$$

Чтобы найти $x_{1}$, проинтегрируем выражение (16):

$$
x_{1}=\int_{0}^{t} \frac{F-K \mu}{m_{1}} t d t+\int_{0}^{t} \frac{K \mu}{m_{1} k_{2}} \sin k_{2} t d t+\int_{0}^{t} C_{1} d t .
$$

Получим

$$
x_{1}=\frac{F-K \mu}{m_{1}} t^{2}-\frac{K \mu}{m_{1} k_{2}^{2}} \cos k_{2} t+C_{1} t+C_{2},
$$

где $C_{1}$ и $C_{2}$ - постоянные интегрирования.

Для определения постоянных интегрирования зададимся начальными условиями: $\dot{x}_{1}=V_{s}$, так как в начальный момент времени тело $m_{1}$ двигалось со скоростью $V_{s} ; x_{1}=0 ; t=0$.

Таким образом, подставив начальные условия в выражения (16) и (18), получим

$$
C_{1}=V_{s} ; C_{2}=-\frac{K \mu}{m_{1} k_{2}^{2}} \text {. }
$$

Деформация внешней пружины

$$
x_{1}=\frac{F-K \mu}{m_{1}} t^{2}+V_{s} t+\frac{K \mu}{m_{1} k_{2}^{2}}\left(1-\cos k_{2} t\right) .
$$

Шаг витка пружины $t=0,007 \mathrm{M}$, скорость подачи $V_{s}=5 \mathrm{~m} /$ мин, частота колебаний $k_{2}=430 \mathrm{c}^{-1}$, масса оправы $m_{2}=0,275$ кг.

$$
\begin{gathered}
x_{1}=\frac{71-3484 \cdot 0,042}{0,275} 0,007^{2}+5 \cdot 0,007+ \\
+\frac{3484 \cdot 0,042}{0,275 \cdot 430^{2}}(1-\cos 430 \cdot 0,007)=0,027 \mathrm{M} .
\end{gathered}
$$
жения

Тогда углубление сверла $z_{2}$ найдем из выра-

$$
z_{2}=x_{1}-x_{2} .
$$

Подставив (13) и (17) в (20), получим

$$
\begin{gathered}
z_{2}=\frac{F-K \mu}{m_{1}} t^{2}+V_{s} t+ \\
+\frac{K \mu}{m_{1} k_{2}^{2}}\left(1-\cos k_{2} t\right)-\frac{K}{c_{1}} \mu\left(1-\cos k_{2} t\right) .
\end{gathered}
$$

Преобразовав выражение (21) с учетом (11), получим

$$
z_{2}=\frac{F-K \mu}{m_{1}} t^{2}+V_{s} t+\left(1-\cos k_{2} t\right)\left(\frac{K \mu^{2}}{m_{1} c_{1}}-\frac{K \mu}{c_{1}}\right) \text {. }
$$

Подставив численные значения в формулу (22), получим углубление сверла:

$$
\begin{aligned}
& z_{2}=\frac{70-3483 \cdot 0,042}{0,275} \cdot 0,0157^{2}+5 \cdot 0,0157+ \\
& \quad+(1-\cos 283 \cdot 0,0157) \times \\
& \times\left(\frac{3484 \cdot 0,042^{2}}{0,275 \cdot 3365}-\frac{3484 \cdot 0,042}{3365}\right)=0,011 \mathrm{M} .
\end{aligned}
$$

Однако выражение (22) довольно сложно оптимизировать, поэтому расчет будем вести с той позиции, что сила $F$ является постоянной. Тогда $x_{1}=V_{s} t \dot{x}_{1}=V_{s}, \ddot{x}_{1}=0 ; t=0$.

$$
\begin{gathered}
m_{2}\left(\ddot{x}_{1}-\ddot{x}_{2}\right)=c_{2} x_{2}-F ; \\
m_{2} \ddot{x}_{2}+c_{2} x_{2}=F,
\end{gathered}
$$

где $c_{2}-$ коэффициент жесткости внутренней пружины, Н/м.

$$
c_{2}=\frac{G \cdot d_{D}^{4}}{8 \cdot d_{F}^{3} \cdot n} .
$$

Модуль сдвига $G=82$ ГПа для стали 60С2А. Диаметр проволоки $d_{D}=0,0006$ м, диаметр завивки $d_{F}=0,0054$ м, Число витков $n=12$.

$$
c_{2}=\frac{82 \cdot 10^{9} \cdot 0,0006^{4}}{8 \cdot 0,0054^{3} \cdot 12}=703,01 \mathrm{H} / \mathrm{M} .
$$

Произведем замену

$$
k_{20}^{2}=\frac{c_{2}}{m_{2}} .
$$

Коэффициент жесткости внутренней пружины $c_{2}=703 \mathrm{H} / \mathrm{M}$, масса сверла $m_{2}=0,05$ кг.

$$
k_{20}^{2}=\frac{703,1}{0,05}=14060,2 \mathrm{c}^{-1} \text {. }
$$

Также представим $F$ как

$$
F=\frac{F_{0}}{h}\left(V_{s} t-x_{2}\right),
$$

где $h$ - биссектриса угла заточки сверла, м. 
Разделим правую и левую части на $m_{2}$ и с учетом (26) и (27) получим

$$
\ddot{x}_{2}+k_{20}^{2} x_{2}=\frac{F_{0}}{m_{2} h}\left(V_{s} t-x_{2}\right) .
$$

Для облегчения расчетов произведем замену:

$$
k_{2}^{2}=k_{20}^{2}+\frac{F_{0}}{m_{2} h} .
$$

Осевая сила $F_{0}=159,07 \mathrm{H}$, биссектриса угла заточки $h=5$ мм.

$$
\begin{gathered}
k_{2}^{2}=14060,2+\frac{159,07}{0,05 \cdot 0,005}=652860,2 \mathrm{c}^{-1} ; \\
k_{2}=\sqrt{652860,2}=808 \mathrm{c}^{-1} .
\end{gathered}
$$

Тогда выражение (28) примет вид

$$
\ddot{x}_{2}+k_{2}^{2} x_{2}=\frac{F_{0} V_{s}}{m_{2} h} t .
$$

Решением выражения (30) будет являться

$$
x_{2}=B \sin k_{2} t+\frac{F_{0} V_{s}}{m_{2} h k_{2}^{2}} t .
$$

Для определения констант $A$ и $B$ продифференцируем выражение (31).

$$
\dot{x}_{2}=B k_{2} \cos k_{2} t+\frac{F_{0} V_{s}}{m_{2} h k_{2}^{2}} .
$$

Начальные условия: $\dot{x}_{2}=0 ; x_{2}=0 ; \mathrm{t}=0$, тогда

$$
B=-\frac{F_{0} V_{s}}{m_{2} h k_{2}^{3}} .
$$

Выражение (32) примет вид

$$
\begin{gathered}
x_{2}=\frac{F_{0} V_{s}}{m_{2} h k_{2}^{2}} t-\frac{F_{0} V_{s}}{m_{2} h k_{2}^{3}} \sin k_{2} t ; \\
x_{2}=0,0036 \mathrm{~m} .
\end{gathered}
$$

Углубления сверла найдем по формуле (5) с учетом (18) и условий работы системы. Получим

$$
z_{2}=V_{s} t-\frac{F_{0} V_{s}}{m_{2} h k_{2}^{2}} t+\frac{F_{0} V_{s}}{m_{2} h k_{2}^{3}} \sin k_{2} t .
$$

Произведем замену

$$
\frac{F_{0}}{m_{2} h}=k_{2}^{2}-k_{20}^{2}
$$

Тогда

$$
z_{2}=\frac{k_{20}^{2}}{k_{2}^{2}} V_{s} t+\left(1-\frac{k_{20}^{2}}{k_{2}^{2}}\right) \frac{V_{s}}{k_{2}} \sin k_{2} t .
$$

Продифференцировав выражение (36) с учетом (32) получим

$$
z_{2}=\frac{k_{20}^{2}}{k_{2}^{2}} V_{s} t+\frac{V_{s}}{k_{2}} \sin k_{2} t ;
$$

$$
\begin{aligned}
& z_{2}=\frac{14060,2}{652860,2} \cdot 5 \cdot 0,002+ \\
& +\frac{5}{808} \sin (808 \cdot 0,002)=0,0064 \mathrm{M} .
\end{aligned}
$$

Как видно из выражения (37), при углублении сверла на $z_{2}$ скорость будет уменьшаться. Однако для точного расчета необходимы более детальные исследования.

Заключение. Согласно полученным данным, глубина сверления будет относительно малой при использовании пружин с коэффициентами жесткости внешней $c_{1}=7797,1 \mathrm{H} /$ м и внутренней $c_{2}=703 \mathrm{H} /$ м пружин. Соответственно, необходимо в дальнейшем исследовать другие упругие элементы для применения их в проектируемом сверлильном инструменте.

Использование данного типа инструмента при сверлении плитных материалов позволит в значительной мере сократить количество брака, увеличить производительность вместе со снижением энергопотребления за счет того, что при помощи упругих элементов будет независимо изменяться скорость подачи инструмента. Предполагаемая стоимость изготовления подобного сверла при серийном производстве значительно меньше затрат на устранение брака или модернизацию используемого оборудования.

\section{Список литературы}

1. Волынский В. Н. Технология древесных плит и композитных материалов: учеб.-справ. пособие. СПб.: Изд-во «Лань», 2010. 336 с.

2. Бершадский А. Л. Расчет режимов резания древесины. Минск: Вышэйшая школа, 1966. 176 с.

3. Цуканов Ю. А., Амалицкий В. В. Обработка резанием древесностружечных плит. М.: Лесная пром-сть, 1966. $94 \mathrm{c.}$

4. Любченко В. И. Резание древесины и древесных материалов: учеб. пособие. М.: Лесная промсть, 1986. 296 с. 
5. Аникеенко А. Ф., Гришкевич А. А., Гаранин В. Н. Влияние элементов режима сверления ламинированных древесностружечных плит на качество поверхности // Труды БГТУ. Сер. 1, Лесное хозяйство, природопользование и переработка возобновляемых ресурсов, 2017. № 2 (198). С. 391-394.

6. Пружины сжатия. Конструкция и размеры: ГОСТ 18793-80. Введ. 17.12.1992. Минск: Гос. ком. по стандартизации Респ. Беларусь, 1992. 88 с.

7. Сверлильный инструмент. URL: https://www.leuco.com/RU/RU/Boring_Bits (дата обращения: 22.03.2021).

8. Сверла. URL: https://leitz.by/index.pl?act=SECTION\&section=sverla (дата обращения: 22.03.2021).

9. Сверла. URL: https://faba78.ru/products/category/sverla (дата обращения: 22.03.2021).

10. Сверла для присадочных станков. URL: http://freud-catalog.ru/catalog/376/692/index.htm (дата обращения 22.03.2021).

11. Сверла для деревообработки. URL: https://www.stanki.by/catalog/svyerla/ (дата обращения 22.03.2021).

12. Сверла спиральные дереворежущие. Технические условия: ГОСТ 22057-76. Введ. 01.01.1978. М.: Изд-во стандартов, 1978. $21 \mathrm{c}$

13. Сверла спиральные с цилиндрическим хвостовиком. Технические условия: ГОСТ 10902-77*. Введ. 01.01.1979. М.: Изд-во стандартов, 1979. 23 с

14. Грубе А. Э. Дереворежущие инструменты: учеб. пособие. М.: Лесная пром-сть, 1971. 344 с.

15. Глебов И. Т. Обработка древесины на станке с ЧПУ: учеб. пособие. Екатеринбург: УГЛТУ, 2018. $142 \mathrm{c}$.

\section{References}

1. Volynskiy V. N. Tekhnologiya drevesnykh plit i kompozitnykh materialov [Board technology and composite materials]. St. Petersburg, Lan' Publ., 2010. 336 p.

2. Bershadski A. L. Raschet rezhimov rezaniya drevesiny [Computation of cutting wood]. Minsk, Vysheyshaya shkola Publ., 1966. 176 p.

3. Tsukanov Yu. A., Amalihskiy V. V. Obrabotka rezaniyem drevesnostruzhechnykh plit [Chipboard cutting]. Moscow, Lesnaya promyshlennost' Publ., 1966. 94 p.

4. Lyubchenko V. I. Rezaniye drevesiny i drevesnykh materialov [Cutting wood and wood material]. Moscow, Lesnaya promyshlennost' Publ., 1986. 296 p.

5. Anikeenko A. F., Grishkevich A. A., Garanin V. N. The influence of the elements of the mode of drilling laminated chipboard on the surface quality. Trudy BGTU [Proseedings of BSTU], issue 1, Forestry. Nature Management. Processing of Renewable Resources, 2017, no. 2 (198), pp. 391-394 (In Russian).

6. GOST 18793-80. Pruzhiny szhatiya. Konstruktsiya i razmery [Compression springs. Design and dimensions]. Minsk, Standartinform Publ., 1992. 88 p. (In Russian).

7. Sverlil'nyy instrument [Drilling tools]. Available at: https://www.leuco.com/RU/RU/Boring_Bits (accessed 22.03.2021).

8. Sverla [Drills]. Available at: https://leitz.by/index.pl?act=SECTION\&section=sverla (accessed 22.03.2021).

9. Sverla [Drills]. Available at: https://faba78.ru/products/category/sverla (accessed 22.03.2021).

10. Sverla dlya prisadochnykh stankov [Drill bits for filler machines]. Available at: http://freud-cata$\log$. ru/catalog/376/692/index.htm (accessed 22.03.2021).

11. Sverla dlya derevoobrabotki [Drills for woodworking]. Available at: https://www.stanki.by/cata$\log /$ svyerla/ (accessed 22.03.2021).

12. GOST 22057-76. Sverla spiral'nyye derevorezhushchiye. Tekhnicheskiye usloviya [Wood-cutting spiral drills. Specifications]. Moscow, Izdatel'stvo standartov Publ., 1978. 21 p. (In Russian).

13. GOST 10902-77*. Sverla spiral 'nyye s tsilindricheskim khvostovikom. Tekhnicheskiye usloviya [Spiral drills with a cylindrical shank. Specifications]. Moscow, Izdatel'stvo standartov Publ., 1977. 23 p. (In Russian).

14. Grube A. E. Derevorezhushchiye instrumenty [Woodworking tools]. Moscow, Lesnaya promyshlennost' Publ., 1971.344 p.

15. Glebov I. T. Obrabotka drevesiny na stanke s CHPU [Wood processing on a CNC machine]. Yekaterinburg, UGLTU Publ., 2018. 142 p.

\section{Информация об авторах}

Аникеенко Андрей Федорович - кандидат технических наук, доцент кафедры деревообрабатывающих станков и инструментов. Белорусский государственный технологический университет (220006, г. Минск, ул. Свердлова, 13а, Республика Беларусь). E-mail: dosy@belstu.by 
Машорипова Татьяна Александровна - аспирант кафедры деревообрабатывающих станков и инструментов. Белорусский государственный технологический университет $(220006$, г. Минск, ул. Свердлова, 13a, Республика Беларусь). E-mail: t.a.mashoripova@mail.ru

\section{Information about the authors}

Anikeenko Andrey Fedorovich - PhD (Engineering), Assistant Professor, the Department of Woodworking Machines and Tools. Belarusian State Technological University (13a, Sverdlova str., 220006, Minsk, Republic of Belarus). E-mail: dosy@belstu.by

Mashoripova Tatiana Aleksandrovna - $\mathrm{PhD}$ student, the Department of Woodworking Machines and Tools. Belarusian State Technological University (13a, Sverdlova str., 220006, Minsk, Republic of Belarus). E-mail: t.a.mashoripova@mail.ru 